\title{
FACTORES QUE INFLUYEN EN LA ADOPCIÓN DE TECNOLOGÍAS DE CONSERVACIÓN DE SUELOS Y AGUA EN LA COMUNIDAD DE TOMABÚ, MUNICIPIO DE LA TRINIDAD ESTELÍ, 2002-2006
}

\section{Fidel Guzmán Guillén', Oswaldo Pineda Rizo', Jeremy Reyes Vallejos ${ }^{2}$, Kevin Aráuz Rodríguez ${ }^{\mathbf{2}}$}

${ }^{1}$ Ing. Agr. MSc. Departamento de Desarrollo Rural. UNA. km 12 1/2 Carretera Norte, Managua, Nicaragua. E-mail: fidel.guzman@una.edu. ni Teléf.- 2233-1999- Tele Fax: 2233-1968

${ }^{1}$ Ing. Agr. Departamento de Desarrollo Rural. UNA. opineda621@gmail.com

${ }^{2}$ Egresados de la Facultad de Desarrollo Rural. UNA

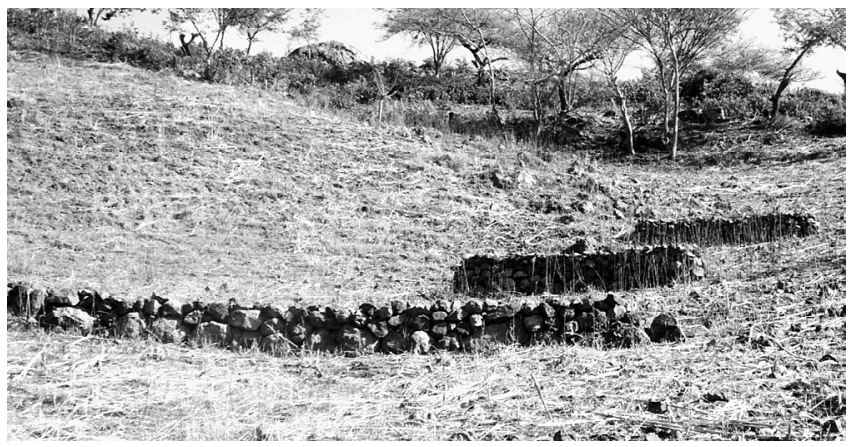

\section{RESUMEN}

El estudio fue realizado en la Comunidad Tomabú del Municipio de La Trinidad Estelí en el período 2002-2006. En el estudio se definió una muestra propositiva y de carácter opinático, Abarcó a productores que cumplieran los siguientes criterios: área entre 0.5 a menos, hasta $10 \mathrm{Mz}^{1}$, de 10.01 hasta $50 \mathrm{Mz}$ y de 50.01 hasta $100 \mathrm{Mz}$. Otro criterio empleado en la muestra razonada es que fuesen productores que hayan sido atendidos por instituciones, productores que cultiven granos básicos y hortalizas y que hayan recibido capacitación y asistencia técnica en obras de conservación de suelos y agua. Considerando esos criterios, hace un total de 28 productores correspondiente al $48.3 \%$ de los hogares existentes en la comunidad. El estudio se centró en cuatro variables que son: Capacitación y Asistencia técnica, Tenencia de la tierra, Ingresos familiares y Organización. Para ello se hizo una revisión bibliográfica, se aplicaron entrevistas, talleres participativos y observación. Los resultados muestran que El nivel de escolaridad fue uno de los factores que influyó en el nivel de adopción, ya que se encontró que los mayores niveles de adopción los presentan productores con mayores niveles de escolaridad (46\% primaria, 28\% secundaria, $9 \%$ estudios universitarios). Los productores que no saben leer ni escribir adoptaron al menos una de las TCSyA. Entre los principales incentivos otorgados por el Instituto Nicaragüense de Tecnología Agropecuaria (INTA), La Unión Nacional de Agricultores y Ganaderos (UNAG), la Fundación para la

\begin{abstract}
The present study was carried out at Tomabú community, municipality of La Trinidad Estelí, during the period extended from 2002 to 2006 . The sample includes farmers who fulfilled the following criteria: area between 0.5 to less up to $10 \mathrm{Mz}$, from 10.01 up to $50 \mathrm{Mz}$ and from 50.01 up to $100 \mathrm{Mz}$. Another criterion used in the reasoned sample were to include farmers that have been attended by institutions, farmers who cultivate basic grains and vegetables and who have received training and technical assistance in soil and water conservation and water practices. From these criteria, the sample was formed by 28 farmers, which correspond to $48.27 \%$ of the existing homes in the community. The study focused on four variables: training and technical assistance, ownership of the land, income and organization. The study started with a literature reviewed, interviews were applied, participative workshops and observation. The results show that educational level was one of the factors that have major influence on level of adoption. The higher levels of adoption were found on farmers with higher levels of education $(46 \%$ primary, $28 \%$ secondary, $9 \%$ university degree). Producers who can not read and write took at least one of the TCSyA. The results show that the principal incentives granted by the Nicaraguan Institute of Agricultural Technology (INTA), the National Union of Farmers (UNAG), the Foundation for Research and Rural Development (FIDER) and the Association for the Development of the Peoples (ADP) were; food, travel allowances, credits and seeds, which contributed largely that producers adopt the technologies of soil and water conservation (TCSyA). Considering training and
\end{abstract}


Investigación y el Desarrollo Rural (FIDER) y la Asociación para el Desarrollo de los Pueblos (ADP) fueron; alimentos, viáticos, créditos y semillas, los cuales que contribuyeron en gran parte a que los productores adoptaran las Tecnologías de Conservación de Suelos (TCSyA). De la Capacitación y Asistencia técnica los productores entrevistados fueron capacitados en curvas a nivel y la mayoría de ellos (93\%) adoptó esta tecnología, el 79\% de los capacitados en barreras muertas adoptó el 50\%, el 75\% de los capacitados en barreras vivas adoptó un $43 \%$, en diques del $68 \%$ solo un $4 \%$, en acequias del $54 \%$ solo un 7\%, siendo estas las cinco TCSyA en las cuales lo productores han sido capacitados con mayor frecuencia. Cuando las tierras son alquiladas disminuye la probabilidad de adoptar, los resultados muestran que la mayor cantidad de TCSyA fueron adoptadas por los productores que disponen de tierras propias (99\%), en comparación a los que alquilan tierra. El ingresos total del hogar conformado por ingresos productivos e ingresos extra agrícolas (remesas familiares) tuvo un comportamiento diferente, los $\underline{\text { Ingresos }}$ Económicos no son un factor influyente en la adopción pues tanto los productores que tuvieron balances anuales altos, bajos y hasta negativos, llegaron a adoptar desde una hasta más de 10 técnicas. Aquellos productores que adoptaron mayor cantidad de TCSyA obtuvieron mayores ingresos productivos que los que complementaban con remesas familiares. En lo que respecta al componente Organización los productores organizados presentaron un mayor grado de adopción, pues hubo productores que llegaron a adoptar hasta 16 de las técnicas transferidas en relación a ocho diferentes técnicas adoptadas de los que no están organizados. Finalmente, las variables en estudio, a excepción de los Ingresos Económicos, determinan los niveles de adopción en la zona de estudio. Se requiere por tanto que todo proyecto de desarrollo rural considere estos factores para lograr éxito en su gestión y contribuir eficientemente en la mejora de las condiciones de producción en los sistemas productivos en donde se tiene inferencia.

Palabras clave: Adopción, Conservación de suelo, Tecnología, Extensión technical assistance, farmers were trained on curves of level and the majority (93\%) adopted this technology. Seventy nine percent of farmers trained in dead barriers adopted 50 $\%$, seventy five percent of farmer trained on alive barriers adopted $43 \%$, in dikes of $68 \%$ only $4 \%$, in irrigation ditches of $54 \%$ only $7 \%$ adopted. The mentioned five TCSyA were the technique in which the farmers have been qualified most. When the lands are rented, the probability of adoption is reduced. The results show that the higher amounts of TCSyA's techniques were adopted by farmers that have their own land $(99 \%)$ in comparison to those who rent it. The total income of the farmer family shaped by productive income and extra agricultural income (family remittances) had a different behavior. The economic income is not an influential factor in the adoption, thus, the producers who had annual high, low and up to negatives balances, managed to adopt from one to even more than 10 technologies. However, those producers who adopted major quantity of techniques obtained major productive income than those that were complementing with family remittances. Regarding organization, organized framers presented a major degree of adoption, since there were farmers who managed to adopt up to 16 of the technologies transferred, in relation to eight different adopted technologies of those who were not organized. Finally, with the exception of the economic income, the other variables determine the levels of adoption in the area. It is required that any project of rural development considers those factors to achieve success in management and to contribute efficiently in improvement production conditions in the productive systems.

Keywords: Adoption, soil and water conservation, technology, extension

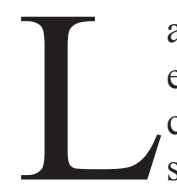

a característica más distintiva de la agricultura es su dinamismo. Las prácticas de cultivo cambian continuamente y sobre la base de su propia experiencia y la de sus vecinos los agricultores perfeccionan la forma en que manejan sus cultivos. Los cambios de las condiciones naturales, la disponibilidad de recursos y el desarrollo de mercados plantean retos y oportunidades a los que responden los agricultores. Además estos se enteran de la existencia de las tecnologías nuevas producidas por diversos organismos, programas, proyectos dedicados a la investigación, la extensión o el desarrollo rural. Estos organismos generan variedades, insumos, prácticas de manejos nuevos y fomentan su empleo, y es esencial que puedan seguir los resultados de sus esfuerzos y comprendan como las tecnologías que promueven encajan en el complejo patrón de cambios en que participan todos los agricultores (CIMMYT, 1993). 
El 80 por ciento de los suelos en Nicaragua se encuentran en laderas, los cultivos alimenticios son producidos por pequeños productores en tierras marginales, por lo que desde la década de los ochentas, los organismos públicos y privados, preocupado por el deterioro creciente de los recursos naturales principalmente el suelo, han promovido tecnologías de conservación de suelos y agua para incrementar la producción, conservando y protegiendo los recursos naturales, como una alternativa al problema de la erosión del suelo que se da principalmente en zonas de laderas.

Los suelos presentan incongruencia entre su uso potencial y actual, siendo los municipios que presentan el mejor uso de la tierra Estelí (36.3\%) y Pueblo Nuevo $(27.5 \%)$. En cambio el resto presentan por encima del $50 \%$ de sus territorios degradados por sobre utilización, sobresaliendo San Nicolás y San Juan de Limay con 70.2 $\%$ y $61.3 \%$ respectivamente. La mayor sub utilización corresponde a La Trinidad con $43.8 \%$. Los municipios que presentan los recursos naturales más degradados que limitan su desarrollo económico son San Nicolás, San Juan de Limay y La Trinidad. Dadas las características predominantes de la región en cuanto a topografía accidentada y suelos de textura franco arenosas hacen que este territorio sea muy vulnerable a los procesos de erosión hídrica de los suelos. (SINAPRED, 2004).

Unos de los principales problemas en la transferencia de Tecnología de Manejo Sostenible de Suelo y Agua (MSSA) en Nicaragua desde los años 70 fue la falta de indicadores que permitieran poder medir su calidad de establecimiento, así como la estrategia de comunicación durante el proceso de aceptación y adopción por parte los agricultores (IICA, 2005).

La adopción de técnicas de conservación de suelos y aguas (TCSyA) por las familias campesinas de la pequeña y mediana producción es el objetivo de muchos organismos que promueven la agricultura sostenible en Nicaragua. Muy a menudo se afirma que esta adopción es lenta y que el proceso de adopción es complejo, sin embargo no se cuenta con mucha información sobre este proceso (AT\&V/PASOLAC, 1999). Rhoda (2005), refieren que la inercia de decisión es descrita como un estado intermedio en el proceso de difusión y agregan que este estado ocurre cuando un individuo desarrolla una actitud neutra sobre la innovación y/o decide ni explícitamente adoptar, ni explícitamente rechazar la innovación.

La adopción depende no solo de la buena voluntad de los agricultores, también depende de los derechos de propiedad, derecho a los recursos y a la acción de comunidad colectiva. (Perret \& Stevens, 2006) Varias instituciones gubernamentales y no gubernamentales han invertido gran cantidad de recursos económicos, materiales y técnicos en proyectos de transferencia de tecnologías de conservación de suelos y agua en algunos de los municipios y comunidades de nuestro país, en donde a menudo se encuentra un fenómeno que obstaculiza la factibilidad de dichos proyectos.

El fenómeno es el bajo nivel de adopción de las tecnologías transferidas a los productores por las instituciones presentes en las zonas donde laboran. Si las instituciones no cuentan con información que les permita identificar cuales son esos factores o elementos que dificultan el proceso de adopción no podrán mejorar sus estrategias de transferencia, pues no sabrían sobre que elementos deben de incidir, pues estos rara vez reservan fondos para monitorear el progreso, realizando estudios de adopción que permitan conocer la cantidad de agricultores que continúan haciendo las practicas promovidas o bien han adecuado estas a sus sistemas de producción cuando el período del proyecto ha concluido (AT\&V/PASOLAC, 1999).

Debido a lo anterior se realizo un estudio para identificar cuales son los factores que determinan el grado de adopción de tecnologías de conservación de suelos y agua (TCSA) transferidas por diferentes instituciones a los productores de la comunidad de Tomabú, Municipio de la Trinidad, Departamento de Estelí en el período 2002-2006.

\section{MATERIALES Y MÉTODOS}

Ubicación del área de estudio. La localización geográfica de la comunidad rural de Tomabú se ubica en el Municipio de La Trinidad, Departamento de Estelí, a una distancia de 15 kilómetros al sur de la cabecera departamental Estelí, y queda comprendida entre las coordenadas geográficas siguientes: Latitud 14³9' a $14^{\circ} 40^{\prime}$ Norte y Longitud 577605 - 579606. La topografía que presenta la comunidad es completamente irregular, siendo su ubicación rodeada de laderas, con áreas montañosas.

El clima que presenta es del tipo sabana tropical modificado de conformidad con la clasificación de W. Koppen. Encontrándose en el sector de arriba con un clima semi húmedo por encontrarse en la zona de amortiguamiento de la reserva forestal Tomabú. Las temperaturas medias anuales se sitúan en $\operatorname{los} 24^{\circ} \mathrm{C}$ a $25^{\circ} \mathrm{C}$. Durante los meses secos (Marzo - Abril) se pueden presentar temperaturas del orden de $\operatorname{los} 29^{\circ} \mathrm{C}$ como promedio, siendo estos posiblemente los meses más calurosos del año. Según registros de la estación metereológica, la humedad relativa del aire alcanza un 
valor medio de 75\%. Existen la época seca y la época lluviosa. La época seca, transcurre de noviembre hasta los primeros 15 días de mayo, se denomina verano.

El período lluvioso comienza a mediados de mayo y termina en noviembre. La precipitación promedio anual es de 873 - $1000 \mathrm{Mm}$. anuales., registrándose la mayor pluviosidad, durante los meses de Junio, Septiembre y octubre, período en el cual ocurre el 94\% de la precipitación media anual. Según registros del MAGFOR Estelí y de la estación meteorológica de la montaña de INETER. El mes de mayor pluviosidad es el de Octubre, en el cual se registra un promedio de 4200 $\mathrm{mm}$. A partir de diciembre hasta abril, las precipitaciones son reducidas.

La Vegetación mas abundante es de pinares asociados con robles encinos del tipo Nebliselva en las partes altas de la cabecera de la comunidad (Cerro Tomabú).

El área de estudio se localiza en la provincia Geomorfológico tierra alta del interior, (Región montañosa del interior), en las mesas escalonadas de Estelí mas conocidas como las mesas de Estelí. (MAGFOR, 2008)

\section{Proceso metodológico}

\section{Fase preliminar o de organización}

a) La recopilación de diversas fuentes bibliográficas

b) Definición de las variables a medir: Estas son Tenencia y uso de la tierra, Asistencia técnica y capacitación, Organización e Ingresos Económicos

c) Primera visita de campo: La idea es de darnos un panorama de la situación actual de los actores definidos en el estudio y se realizaron observaciones de campo.

d) validación de los instrumentos y definición de la muestra: Se realizó una segunda visita de campo para la validación de los instrumentos con los productores de la comunidad en estudio. Empleamos la muestra razonada también conocida como muestra opinática, es decir, elegimos casos que son considerados típicos de la población de interés (Kashif H. \& Erdogan H. 2007) Para definir la muestra se consultó datos estadísticos del Municipio de la Trinidad, Departamento de Estelí (INEC, 2001) y fue determinada por criterios de selección donde los elegidos cumplieran con algunos requisitos, es decir, aquellos productores de la Comunidad de Tomabú que han sido expuestos a las tecnologías, productores que han sido atendidos por instituciones que han trabajado en técnicas en conservación de suelos y agua, productores con un rango de área entre De 0.5 a Menos hasta 10 Manzanas, de 10.01 hasta 50 Manzanas y de 50.01 hasta
100 Manzanas, Productores que cultivan granos básicos y hortalizas. A partir de esto se abarcó a Personal Técnico y de Dirección de las siguientes instituciones: Instituto Nicaragüense de Tecnología Agropecuaria (INTA), Unión Nacional de Agricultores y Ganaderos (UNAG), VISION MUNDIAL y Fundación Iberoamericana de Desarrollo Rural (FIDER).

Fase central o de ejecución. A partir de la definición de los instrumentos y la muestra definidos en la fase anterior, procedimos a:

a) Aplicación de Entrevistas a productores: Se procedió a la aplicación de las entrevistas a Informantes claves, empleando además la técnica de la Observación directa.

b) Implementación de talleres participativos: Realizamos talleres participativos con actores locales (productores, personal técnico...) en los cual analizamos a través de un proceso valorativo de cada uno de los aspectos o tópicos del estudio.

c) Aplicación de entrevistas a técnicos de instituciones: Visitamos a los técnicos que en su momento laboraron en las diferentes instituciones que trabajaron en la transferencia de tecnologías de conservación de suelo y agua en la comunidad de Tomabú.

d) Procesamiento de la información: Una vez recopilada y ordenada la información empleamos los cuadros de salida y cuadros consolidados diseñados sobre la base de las preguntas o tópicos que contienen los instrumentos, luego empleamos datos de frecuencia y porcentajes de cada variable y de cada actor social el cual se procesó y se analizó haciendo uso de programas de Excel y de Word en ambiente Windows por cada variable de estudio.

Fase final. La realización de esta fase correspondió a:

a) Elaboración del documento preliminar: Una vez procesada la información recopilada en el estudio elaboramos un documento preliminar, en donde reflejamos el comportamiento de cada una de las variables definidas así como la triangulación de éstas, de forma que nos permitiera corroborar nuestra hipótesis planteada.

b) Socialización de la información: Con el documento preliminar Posteriormente procedimos a un proceso de socialización o restitución de la información analizada con los actores locales a través de un Taller participativo con el propósito de corroborar los datos obtenidos y validar las conclusiones preliminares del Estudio. 
c) Elaboración del documento final: Obtenido los comentarios y observaciones a los resultados preliminares del Estudio por parte de los actores locales procedimos a la elaboración del Documento final del proceso investigativo.

\section{RESULTADOS Y DISCUSIÓN}

Estructura de la familia. Las 28 familias entrevistadas suman en total 151 personas, correspondiente al 23.9\% de la población total de la comunidad de 630 habitantes (FISE 2007).

Tabla 1. Estructura de la familia

\begin{tabular}{lccccc}
\hline \multicolumn{1}{r}{ Lactantes } & Niños/as & Hombres & Mujeres & \\
\hline Edad & 0 a 1 año & 2 a 18 años & 19 a $>50$ años & $>50$ años & Total \\
Cant. & 1 & 57 & 51 & 42 & 151 \\
$\%$ & 0,66 & 37,75 & 33,77 & 27,81 & 100 \\
\hline
\end{tabular}

Fuente: Elaboración propia.

Como se observa en la tabla 1, los niños/as conforman el mayor porcentaje en la estructura de la familia y estos están comprendidos entre las edades de 2 a 18 años, los jóvenes en esta edad son potenciales adoptadores para futuros proyectos de transferencias de tecnologías de conservación de suelos y agua. Martínez \& Cruz, 2002 afirman que la edad es una característica examinada en estudios de adopción, puesto que esta puede incidir en la adopción de diversas formas.

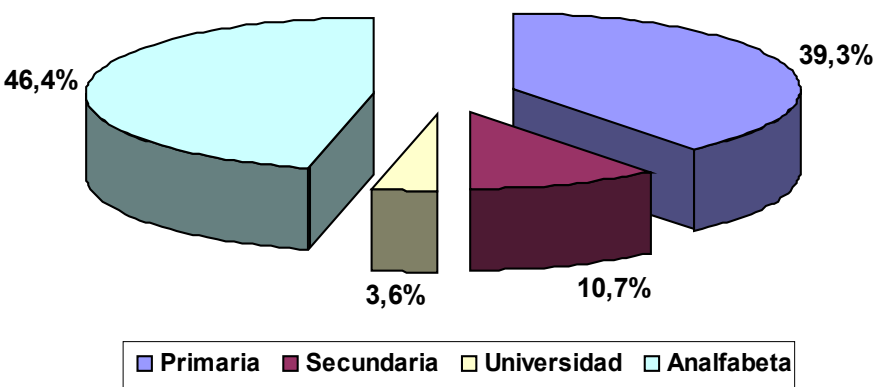

\section{Escolaridad alcanzada}

Figura 1. Escolaridad alcanzada por productores.

Todos de los productores entrevistados son mayores de edad y de acuerdo con un estudio socioeconómico realizado por el FISE 2007, el índice de analfabetismo en las personas adultas es de $18 \%$, esto limita el desarrollo en la gestión comunitaria, lo cual no les permite proyectarse social ni económicamente, lo anterior se suma a la falta de servicios básicos (FISE, 2007).
La figura 1 muestra que el $39.3 \%$ de los productores entrevistados alcanzaron un nivel de escolaridad de primaria, $10.71 \%$ secundaria, el $3.57 \%$ alcanzaron niveles de estudios universitario y $46.43 \%$ son analfabetas. Como se puede observar la proporción porcentual de analfabetismo entre los productores entrevistados es grande, esto se con-vierte en un obstáculo a la hora de la transferencia de tecnologías ya que el proceso de comunicación entre productor-técnico (emisor-receptor) se vuelve más complejo tanto para transmitir el mensaje como para seleccionar el método más adecuado para hacerlo.

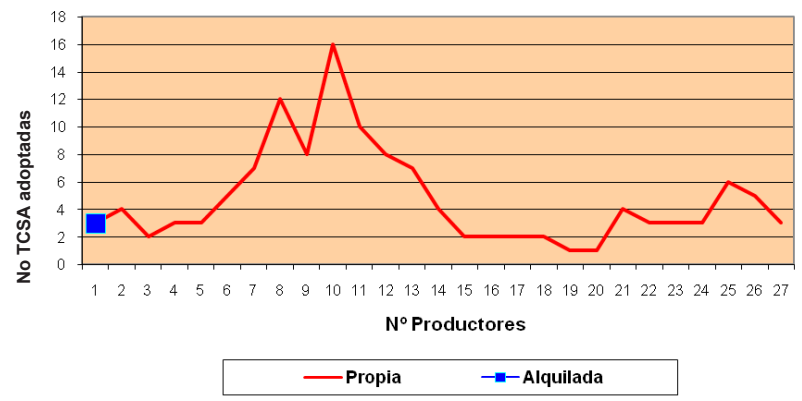

Figura 2. Número de tecnologías adoptadas según escolaridad.

No obstante, si observamos la figura 2 veremos el comportamiento de la adopción según el nivel de escolaridad y notaremos que independientemente del nivel escolar alcanzado por los productores de la zona de estudio, hubo productores sin ningún nivel de escolaridad que adoptaron hasta 8 diferentes tipos de TCSyA.

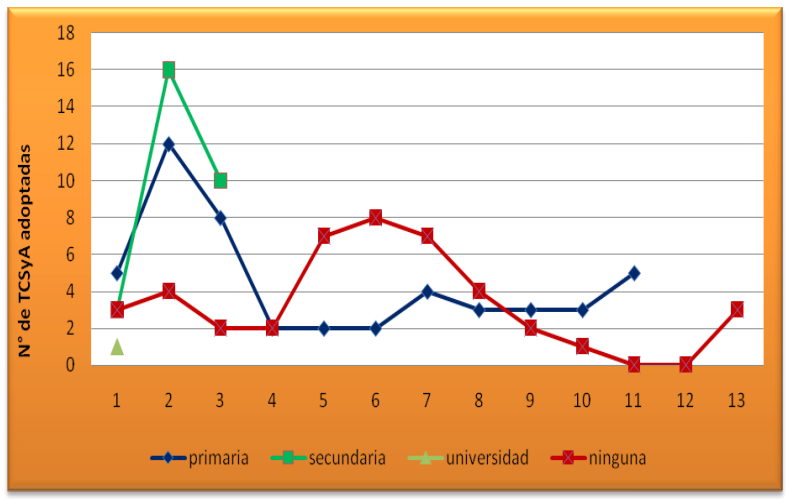

Figura 3. $\mathrm{N}^{0}$ de tecnologías adoptadas según tenencia de la tierra. 


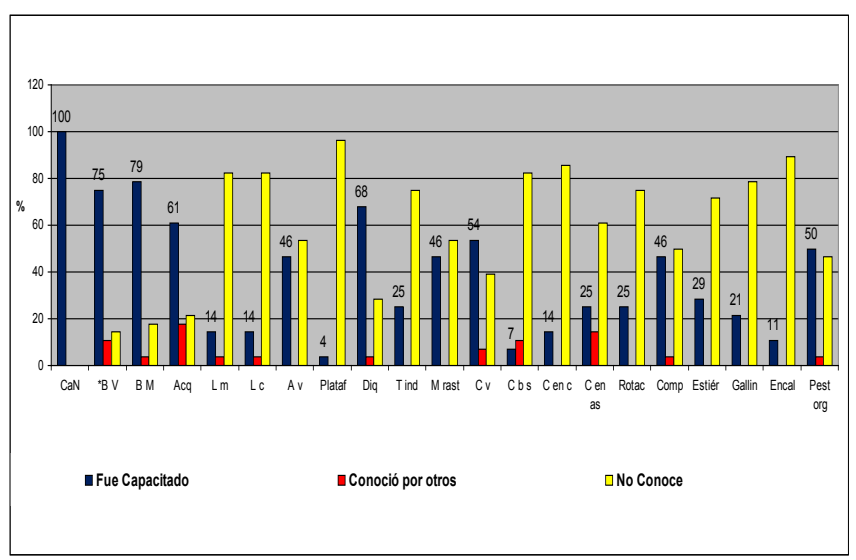

Figura 4. Distribución Porcentual de productores con relación al origen del conocimiento de las TCSyA.

Uso y tenencia de la tierra. La tenencia de la tierra es unos de los factores que considera el productor para la toma de decisión en la adopción de tecnologías que contribuye a mejorar sus parcelas. La figura 3 ilustra el comportamiento que presentaron los dueños $\mathrm{y}$ arrendatario de tierra referente al número de TCSA adoptadas.

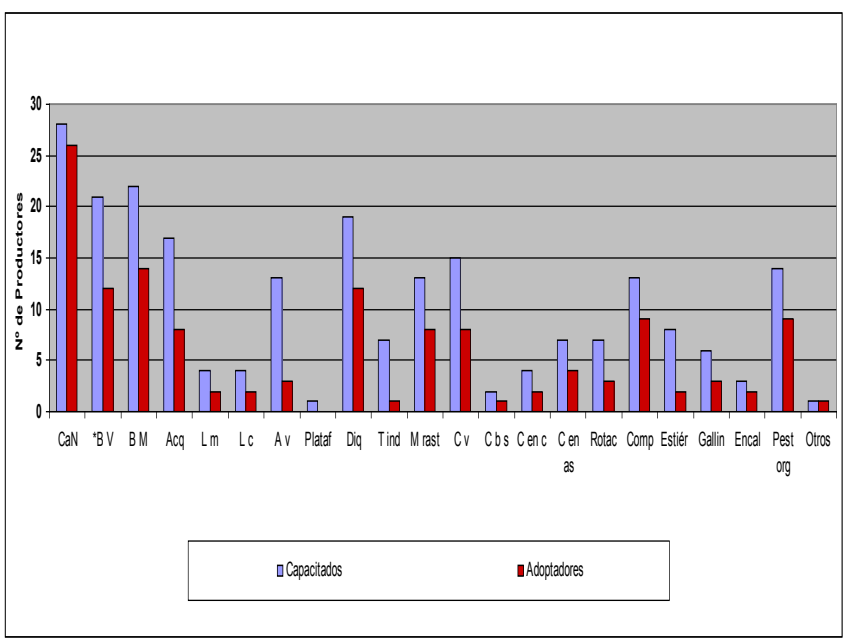

Figura 5. Influencia de las capacitaciones en la Adopción de TCSyA.

Esta relación confirmó que los propietarios suelen estar más dispuestos en adoptar e invertir fuerza de trabajo en técnicas que contribuyen a mejorar la fertilidad de suelo y productividad, para este caso los dueños de propiedades representa el $96 \%$. Por el contrario los que alquilaron tierra para cultivarlas se encuentran menos motivados para este tipo de adopción.
Capacitación y asistencia técnica. Es importante destacar que los Servicios de Extensión encargados de los procesos de transferencia de tecnologías, tienen un fuerte peso en las decisiones de adopción (Yila, O., \& Thapa, G. 2008). Las familias que cultivan de manera convencional pero compartiendo muchas características identificadas con familias sostenibles, potencialmente son aquellos las mejores apuntadas para programas educativos (Salamon, S.; et al. 1997)

Sin embargo es necesario destacar que no todos los productores entrevistados conocieron las técnicas a través del personal técnico, otro origen del conocimiento fue a través de otros (vecinos, promotores, líderes...) pero en menor proporción, a como se observa en la figura 4. Y es además necesario considerar, que los servicios de apoyo para el desarrollo rural deberían enfocar su intervención en convencer primero a los agricultores del problema de la degradación de suelo, antes de capacitarlos sobre prácticas de conservación de suelo (Sidibe, A. 2005)

No todos los productores fueron capacitados en las mismas técnicas, mas bien la participación en los encuentros era selectiva de acuerdo a la técnica que se iba a transferir, considerando otros factores como la motivación de los productores, las condiciones biofísicas (tipos de suelo, pendiente, degradación, vegetación, Etc.) de cada unidad productiva.

Sin embargo hay técnicas en donde se demuestra que mas del $60 \%$ de los productores beneficiados fueron capacitados principalmente en cinco técnicas y/o obras de conservación de suelo y agua. Esto nos dice que estas obras como las Curvas a nivel (CaN), Barreras vivas $(B V)$, Barreras muertas (BM), Diques (Diq) fueron las más adoptadas por los productores, a como se muestra en la figura 5.

De las 21 técnicas que promovieron los organismos e instituciones como FIDER - INTA, a través de días de campo y talleres participativo, solamente hubo una técnica que todos los productores participaron en los encuentros educativos, es decir que todos los beneficiarios $(100 \%)$ en el proyectos recibieron la capacitación que fue la técnica curva a nivel y de estos el 93\% adoptaron esta tecnología, las barreras vivas el $43 \%, 50 \%$ barreras muertas y un $43 \%$ los diques.

Sidibe, A. (2004) afirma que las variables como la educación y la percepción de degradación de suelo son determinantes para la adopción de Diques. Estas son las técnicas con porcentaje de adopción por parte de los beneficiarios en los proyectos de conservación de suelo y agua. En la decisión del tema a capacitar, el 89\% de los entrevistados manifestaron que el técnico decidía la 
técnica a capacitar y apenas un $11 \%$ de los productores se tomaron en cuenta en la selección del tema a impartir tanto teórico como práctico, generalmente un $89 \%$ de los talleres educativos lo realizaron en la escuela de la comunidad o en la casa de un productor.

$96.43 \%$ de los entrevistados afirman haber recibido incentivos por la realización o construcción de las TCSyA durante el proceso de transferencia de las diferentes tecnologías difundidas por las instituciones con presencia en la zona y solo $3.57 \%$ afirman que no recibieron ningún tipo de incentivos durante este proceso. No obstante, que el productor tenga acceso a la información y cuente con programas de apoyo para la inversión inicial en TCSyA, es determinante para que haya mayor nivel de adopción, afirma Bekele, W. \& Drake L. (2003).

Hellin, J., \& Schrader, K. 2003, afirman que los niveles de adopción por los agricultores, son bajas y muchas organizaciones de desarrollo han vuelto a la utilización de incentivos directos, como pagos e $n$ efectivo y alimento por trabajo, para atraer a agricultores. Agregan que la investigación en Centro America muestra que mientras estos incentivos estimulan la puesta en práctica de tecnologías de conservación de suelos, muchos de los agricultores abandonan las tecnologías una vez que los incentivos directos son retirados

Tipos de incentivos recibidos para realizar las TCSyA. Entre los incentivos otorgados por las diferentes instituciones encontramos que $96.43 \%$ de los entrevistados afirman que recibieron alimentos como incentivos para la realización de las TCSyA, 35.71\% de los entrevistados afirman que a cambio de realizar las TCSyA recibían capacitaciones, $28.57 \%$ afirman que recibían crédito como incentivos, $10.71 \%$ recibieron herramientas agrícolas y $3.57 \%$ afirman haber recibido insumos como incentivos por la realización de las TCSyA.

Ingresos económicos. La economía campesina esta compuesta por el sector agropecuario donde el proceso productivo se desarrolla en unidades de tipo familiar, teniendo por objetivo asegurar el bien de la familia, la reproducción de sus condiciones de vida y del trabajo, en otras palabras, la explotación debe generar los medios necesarios para asegurar el sostenimiento biológico de los integrantes de la familia y la satisfacción de sus necesidades (Aguilar, C \& Jiménez, O. 1998).

Margen bruto de la producción. En la Tabla 2 se muestran los resultados de rendimiento de los rubros maíz (Zea mays, L.) y sorgo (Sorghum vulgare P.) y reflejan márgenes brutos promedios negativos, esto indica que estos dos rubros no son rentables para los productores de esta comunidad, pues los costos de producción superan los ingresos obtenidos por la venta de la producción total, esto se debió a los bajos rendimientos obtenidos en ambos rubros $9.3 \mathrm{qq}$ $\mathrm{Mz}^{-1}$ y $4.74 \mathrm{qq} / \mathrm{Mz}$ respectivamente, los cuales son muy inferiores a los rendimientos promedios estimados a nivel nacional de $22.86 \mathrm{qq} \mathrm{Mz}^{-1}$ y $21.9 \mathrm{qq} \mathrm{Mz}^{-1}$. La chiltoma (Capsicum annum, L.) es el rubro que aparece con el margen bruto más alto, esto se debió al precio alcanzado por este producto durante el período de cosecha, el cual fue de C $\$ 300$ por saco, el rendimiento obtenido fue otro factor que contribuyó a esto (300 sacos/Mz), cabe destacar que solo uno de los productores de entre los entrevistados cultivó este rubro. El tomate (Lycopersicum esculentum, Mill) ocupa el segundo lugar en rentabilidad, pues es el segundo rubro con un margen bruto alto, favorecido por los precios de venta (C\$ 120/cajilla), aunque los rendimientos promedios no hayan sido tan buenos (453 cajillas/Mz), pues los productores afirman que los rendimientos promedios de esta comunidad oscilan entre las 800 y 1000 cajillas.

En tercer lugar se encuentra el rubro frijol (Phaseolus vulgaris L.), este presenta un margen bruto anual promedio de C\$23661 los rendimientos promedios de este son de $9.7 \mathrm{qq} \mathrm{Mz}^{-1}$, los cuales son considerados por los productores como rendimientos medios, pues con esta misma variedad (INTA-Ceda) han obtenido rendimientos promedios de $30 \mathrm{qq} \mathrm{Mz}^{-1}$. Según la consideración del MAGFOR este rendimiento (9.7 qq Mz-1) es aceptable, pues en el informe anual de ese mismo año afirma que los rendimientos promedios

para esta zona son de 9.6 qq/Mz (MAGFOR, 2008).

Tabla 2. Margen bruto anual promedio obtenido por rubro

\begin{tabular}{cccccc}
\hline Rubro & Maíz & Frijol & Sorgo & Tomate & Chiltoma \\
\hline MBA C\$ & -2803 & 23661 & -1546 & 26404 & 103760 \\
& & & & & \\
MBA US\$ & -143.01 & 1207.19 & -78.88 & 1347.14 & 5293.88 \\
\hline
\end{tabular}

Fuente. Elaboración propia.

Organización. Para analizar la influencia de la organización en el número de TCSyA adoptadas se ha dividido a los productores en dos grupos: productores que han estado organizado y productores que no han 
estado organizados, en el presente estudio encontramos que el $67.86 \%$ de los entrevistados ha estado organizado en diferentes grupos y $32.14 \%$ no han pertenecido a ninguno. Entre el grupo organizado tenemos; El comité de la iglesia católica, el grupo de trabajo de la UNAG, el comité de vigilancia, grupo de trabajo de FIDER, grupos de trabajo del INTA. En la figura 6 se observa a productores que han estado organizados (68\% de los entrevistados) son los que han adoptado un mayor numero de TCSyA, salvo en algunos casos en donde aún estando organizados algunos productores adoptaron un numero menor de TCSyA que los que no han pertenecido a ninguna de la estructura organizativas existentes en la comunidad ( $32 \%$ de los entrevistados).

Los productores han afirmado que estos grupos organizacionales en los que han estado afiliados no han tenido ninguna influencia en la adopción de tecnologías, pues las actividades que realizan dentro de estos son ajenas a los proyectos de transferencia y adopción de TCSyA.

Para impulsar formas organizativas, debe tenerse presente que las posibilidades de establecerlas serán menores, mientras menor sean las formas participativas de gestión y mayor grado de heterogeneidad de los intereses de los participantes.

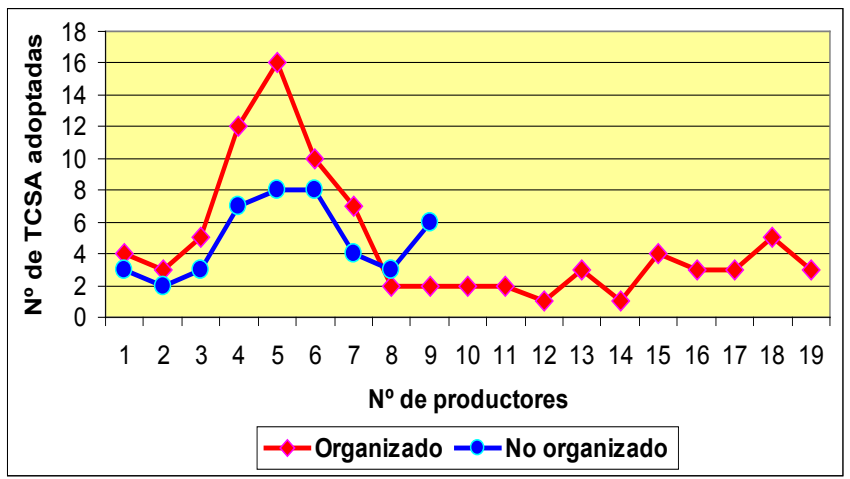

Figura 6. $\mathrm{N}^{\mathrm{o}}$ de tecnologías adoptadas según participación en organización.

Razones por las cuales abandonaron las TCSyA. En el estudio se encontró que al $82.1 \%$ de los entrevistados no se les presentó ningún problema al momento de realizar las prácticas, no obstante algunos productores consideran haber abandonado las TCSA, por diversas razones, tales como la ausencia de acompañamiento de técnicos en el $17.86 \%$ de los casos, otro $17.86 \%$ afirma haber abandonado las TCSyA porque demandan mucha mano de obra, un $14.29 \%$ por que llevan mucho trabajo, el $7.14 \%$ por falta de agua. Además se señala que el campesino aunque quisiera adoptar una nueva tecnología sea acogida, por ejemplo, que un producto no esté disponible a tiempo, que no alcance el dinero o la mano de obra en el momento oportuno, o que no tengan conocimientos adecuados.

\section{CONCLUSIONES}

El nivel de escolaridad es un factor que influye en el nivel de adopción de TCSyA ya que se encontró que los mayores niveles de adopción los presentan aquellos productores con mayores niveles de escolaridad.

El tipo de tenencia de la tierra influyó en el nivel de adopción de TCSyA de los productores de la comunidad de Tomabú ya que presentan mayores niveles de adopción los productores con tenencia propia de la tierra que aquellos que alquilan esta para realizar las actividades productivas.

La afiliación a un grupo organizacional es otro de los factores que influyen en el nivel de adopción de los productores de la comunidad de Tomabú ya que los productores que han estado organizados presentan mayor número de TCSyA adoptadas que aquellos que no han estado organizados.

La recepción del servicio de asistencia técnica también es otro de los factores que influyen en la adopción de los productores de la comunidad de Tomabú puesto que los que han recibido este servicio adoptan más TCSyA que aquellos que no lo han recibido.

Un alto nivel de ingresos económicos en los productores de la comunidad de Tomabú no es un factor determinante en la adopción de TCSyA ya que se encontró que tanto productores con los mayores niveles de ingresos económicos como los menores niveles de ingresos, adoptan la TCSyA.

La opinión de los productores de la comunidad de Tomabú coincide con la de los técnicos que trabajaron con las instituciones en la transferencia de TCSyA al afirmar que las curvas a nivel, barreas vivas y barreras muertas son las TCSyA más adoptadas por los productores de esta comunidad.

\section{RECOMENDACIONES}

Las instituciones interesadas en la ejecución de proyectos de transferencia de TCSyA en la comunidad de Tomabú deben de considerar la escolaridad, la calidad de la asistencia técnica, el nivel de organización y la tenencia de la tierra como determinantes para que los productores adopten una TCSyA.

La selección de los temas de capacitación y las TCSyA que serán transferidas deberán ser seleccionadas de forma participativa por los productores de la 
comunidad, de modo que el técnico funcione como un facilitador de los procesos de cambios tecnológicos.

Las TCSyA que vayan a ser transferidas por las instituciones deberán de ser compatibles con las características socioeconómicas, productivas y ambientales de los productores de esta zona, además deberán de ser fruto de una demanda tecnológica por parte de los productores y no de una oferta brindada por dichas instituciones.

Incorporar en los procesos de transferencia de las TCSyA metodologías y canales interpersonales, ya que estos tienen un mayor efecto para persuadir al productor sobre los beneficios de estas.

\section{REFERENCIAS BIBLIOGRÁFICAS}

Aguilar C.W. y Jiménez O. J. 1998. La Escuela de Agricultura Ecológica: U. Yits. En Revista de la Red de Educadores Ambientales del Sureste. Editorial Probatura Península de Yucatán. Mérida, Yucatán. México.

Asociación Tierra y Vida (ATyV/PASOLAC).1999. Herramientas socioeconómicas de seguimiento y evaluación de la transferencia en CSyA. Editorial Ciencias Sociales, Managua, Nicaragua. 171 pp.

Bekele, W. \& Drake, L. 2003. Soil and water conservation decision behavior of subsistence farmers in the Eastern Highlands of Ethiopia: a case study of the Hunde-Lafto area.

CIMMYT, 1993. La adopción de tecnologías agrícolas: Guía para el diseño de encuestas. Programa de economía del CIMMYT, México, D.F. 88 PP.

Fondo de Inversión Social de Emergencia (FISE). 2003. Proyecto: Construcción De Mabe, Letrinas Y Capacitación Comunitaria en la comunidad de Tomabú.

Hellin, J., \& Schrader, K. (2003, October). The case against direct incentives and the search for alternative approaches to better land management in Central America. Agriculture, Ecosystems \& Environment, 99(1-3), 61. Retrieved July 20, 2009, doi:10.1016/S0167-8809(03)00149-X

IICA Nicaragua. 2005. Inventario de Políticas Agroambientales en Nicaragua, descripción de los principales problemas ambientales relacionado con la agricultura, Pagina 8; Tecnología. Managua Nicaragua.

Instituto Nacional de Estadísticas y Censos (INEC). 2001. Censo Nacional Agropecuario.

Joseph, Rhoda C. 2005. An examination of non-adoption and decision inertia: A Web-based perspective. Ph.D. dissertation, City University of New York, United States -- New York. Retrieved July 20, 2009, from ABI/INFORM Global. (Publication No. AAT 3169931).

Kashif Hussain y Erdogan H. Ekiz. 2007. Estudios y perspectivas en turismo ISSN 1851-1732 versión on-line. Estud. perspect. tur. v.16 n.3 Ciudad Autónoma de Buenos Aires jul./sep. .’Percepción de la calidad del servicio de la calidad del servicio en las empresas aéreas del norte de Chipre. Una aplicación del análisis lineal de patrones".

Mendola M. 2007. Agricultural technology adoption and poverty reduction: A propensity-score matching analysis for rural Bangladesh. Food Policy, 32 (3), pp. 372-393.

Ministerio Agropecuario y Forestal (MAGFOR). 2008. Informe Mensual.

Perret, S., \& Stevens, J. (2006, October). Socio-economic reasons for the low adoption of water conservation technologies by smallholder farmers in southern Africa: a review of the literature. Development Southern Africa, 23(4), 461-476. Retrieved July 28, 2009, doi:10.1080/03768350600927193

Salamon, S.; et al. 1997. "Family factors affecting adoption of sustainable farming systems". Journal of Soil and Water Conservation; Jul/Aug 1997; 52, 4; Academic Research Library. pg. 265

Sidibe, A. 2005. Farm-level adoption of soil and water conservation techniques in northern Burkina Faso. Agricultural Water Management, Volume 71, Issue 3, 15 February 2005, Pages 211-224

Sistema Nacional para la Prevención, Mitigación y Atención de Desastres (SINAPRED). 2004. Plan de Respuesta Departamental con Enfoque de Gestión del Riesgo Departamento de Estelí, REGIÓN I.:

Yila, O., \& Thapa, G. (2008). Adoption of agricultural land management technologies by smallholder farmers in the Jos Plateau, Nigeria. International Journal of Agricultural Sustainability, 6(4), 277-288. Retrieved July 16, 2009, from Academic Research Library. (Document ID: 1614828701). 